\title{
Intensive insulin therapy improves endothelial function and microvascular reactivity in young people with type 1 diabetes
}

\author{
V. L. Franklin • F. Khan • G. Kennedy • J. J. F. Belch • \\ S. A. Greene
}

Received: 29 May 2007 / Accepted: 21 September 2007 / Published online: 27 November 2007

(C) Springer-Verlag 2007

\begin{abstract}
Aims/hypothesis Macrovascular disease is an important cause of the increased morbidity and mortality rates associated with type 1 diabetes, and this vascular impairment begins in childhood. The aim of this study was to determine whether introducing intensive diabetes management [intensive insulin therapy (IIT) and 'Sweet Talk' text-messaging support] produces measurable improvements in endothelial function.

Methods One hundred and twenty-six patients fulfilled the eligibility criteria (type 1 diabetes for $>1$ year; on conventional insulin therapy (CIT); aged between 8 and 18 years), of whom 92 enrolled. Patients were randomised to group 1, CIT only $(n=28)$; group 2, CIT and Sweet Talk $(n=33)$; or group 3, IIT and Sweet Talk $(n=31)$. Vascular assessments (including measures of endothelial damage, activation, dysfunction and oxidative stress) and $\mathrm{HbA}_{1 \mathrm{c}}$ were performed at baseline and repeated after 12 months of the study.

Results Glycaemic control deteriorated in patients on CIT, but improved significantly in patients allocated to IIT $(p=0.007)$. IIT was associated with significantly greater improvements in E-selectin $(p<0.0001)$ than CIT (group 1, $p=0.026$ and group 2, $p=0.053)$. Vascular responses to acetylcholine improved in patients on IIT $(p=0.017)$, but not in patients receiving CIT. These changes were all independent of $\mathrm{HbA}_{1 \mathrm{c}}$ level.
\end{abstract}

V. L. Franklin $(\bowtie) \cdot$ S. A. Greene

Maternal and Child Health Sciences, University of Dundee,

Ninewells Hospital and Medical School,

Dundee, UK

e-mail: victoria.franklin@nhs.net

F. Khan · G. Kennedy • J. J. F. Belch

University Department of Medicine, University of Dundee,

Ninewells Hospital and Medical School,

Dundee, UK
Conclusions/interpretation IIT appears to be associated with improvements in vascular markers, independently of changes in $\mathrm{HbA}_{1 \mathrm{c}}$, suggesting that IIT may confer vascular protection in addition to improving glycaemic control.

Keywords Diabetes in childhood - Endothelium .

Macrovascular disease
Abbreviations
Ach acetylcholine
CIT conventional insulin therapy
IIT intensive insulin therapy
SNP sodium nitroprusside

\section{Introduction}

Vascular disease is the major cause of morbidity and mortality in patients with type 1 diabetes [1]. The overall mortality of patients with type 1 diabetes is $50 \%$ within 40 years of diagnosis, compared with only $10 \%$ of a control population. This increased mortality is related to their risk of micro- and macrovascular disease, which occurs at an earlier age $[2,3]$, and probably has its roots in childhood [4]. There is now compelling evidence that optimising glycaemic control with intensive diabetes management reduces the development and progression of microvascular complications [5]. The mechanisms of development of macrovascular disease are complex and multifactorial, and include contributions from conventional adult risk factors such as hypertension and hyperlipidaemia [6].

More recently, attention has focused on the early detection of abnormal physiology that may predict a high risk of vascular disease in type 1 diabetes. Attention has 
been drawn to the role of the endothelium in diabetes, and its interactions with the white blood cell and other mechanisms of oxidative stress [7]. Our group has previously described that endothelial dysfunction is an early event in type 1 diabetes, with abnormalities present in children with type 1 diabetes even when no clinical signs of vascular complications are present [8-10]. Additionally, we have shown that these early changes in endothelial dysfunction in young people are related to glycaemic control and diabetes duration [9].

Intensive diabetes management involves intensive insulin therapy (IIT) with either a multiple daily injection regimen or with a continuous subcutaneous insulin infusion via a pump. IIT is associated with a significant improvement in glycaemic control and a reduction of the development and progression of microvascular complications including retinopathy [5]. However, IIT is successful only when coupled with increased health professional support such as increased clinic visits and frequent telephone contact [5]. This is reflected in current UK guidelines for the management of type 1 diabetes, which advocate that IIT should be delivered as part of a comprehensive support package $[11,12]$. However, increasing traditional contact between health professionals and patients is expensive to deliver within current health service resources. We therefore developed 'Sweet Talk', a novel motivational support network, using text messages to deliver a theoretically guided behavioural intervention to facilitate uptake of IIT in young people. This has been described in detail previously $[13,14]$. Thus, the aims of the current study were to investigate the impact of introducing intensive diabetes management in young people with type 1 diabetes on markers of endothelial function and skin microvascular reactivity, which we have shown previously to be impaired in young people with type 1 diabetes and to be related to glycaemic control [9]. It is known that microvascular abnormalities demonstrable in the skin are related to increased vascular risk $[15,16]$.

\section{Methods}

Participants The Tayside Medical Ethics Research Committee granted approval for this study and recruitment took place between October 2002 and February 2003. All patients $(n=131)$ attending paediatric diabetes clinics in Tayside, Scotland who were aged between 8 and 18 years, had type 1 diabetes for $>1$ year and were on conventional insulin therapy (CIT; two or three daily injections of premixed insulin), were considered for inclusion in the trial. Five patients were excluded from participation because they were thought unsuitable for IIT (two had serious social problems, one had autism, one had severe needle phobia and one was moving away from the area). Informed consent was obtained from the 92 patients who elected to participate in the study and from their parents. No patients were taking vasoactive medication or other therapy, such as statins, thought to affect vascular function. All children in the study over the age of 12 years had been screened regularly for microvascular complications, according to national guidelines [12]. A small number $(<20 \%)$ had background retinopathy only. None had microalbuminuria or hypertension (although $24 \mathrm{~h}$ blood pressure monitoring was not undertaken in this group).

Study protocol A computer-generated concealed allocation sequence (with block randomisation that balanced at 6,12 , 30 and simple randomisation after 30) assigned participants to one of three groups: group 1, a control group continuing with CIT and usual support; group 2, continuing with CIT and receiving the text message behavioural support intervention; and group 3, changing to IIT and receiving the text message behavioural support intervention. The study did not include a group with IIT and usual support, because the literature indicates that introducing IIT without increasing support is unsuccessful [17]. Patients on CIT received two injections of pre-mixed insulin (Mixtard 30; NovoNordisk Copenhagen, Denmark) with or without one injection of insulin aspart to cover the evening meal (Novorapid; NovoNordisk). Patients on ITT received either basal bolus therapy or insulin pump therapy and carbohydrate-counting education. Patients opting for pump therapy used the Medtronic 508 (Minimed, Watford, UK) pump with the rapid-acting insulin aspart. Pump therapy was initiated as an outpatient in half-day group pump start sessions run by the diabetes team. Patients choosing basal bolus therapy used glargine (Lantus; Sanofi-Aventis, Paris, France) as the once-daily basal insulin and insulin aspart as the bolus insulin to cover carbohydrate content of meals. All patients continued with conventional care delivered by a multidisciplinary team including clinics every 3-4 months and access to an emergency hotline. Patients allocated to the Sweet Talk intervention participated in goal setting at clinic visits and received one or two text messages daily containing tips, information and reminders to reinforce their personal diabetes self-management goal.

Biochemical analysis Venous blood was taken from each patient at the beginning and end of the 12 month study period between 0900 and 1100 hours on each occasion to avoid diurnal variation in the measurements. Discomfort was minimised by the use of local anaesthetic cream. $\mathrm{HbA}_{1 \mathrm{c}}$ was analysed by Bayer DCA 2000 (Newbury, UK; normal range $4.2-6.5 \%$ ). A venous blood sample was obtained from the antecubital fossa using a 23 gauge butterfly needle. Citrated blood was placed immediately into ice and 
centrifuged within $30 \mathrm{~min}$ at $1,500 \times \mathrm{g}$ for $15 \mathrm{~min}$ at $4^{\circ} \mathrm{C}$ and aliquots were stored at $-70^{\circ} \mathrm{C}$ for measurement of E-selectin by ELISA (R\&D Systems, Abingdon, UK).

Ten millilitres of blood were collected into EDTA and prepared as before for the measurement of the various lipids. All lipids (cholesterol, HDL, LDL and triacylglycerols) were measured on a centrifugal analyser using products from Randox Laboratories (Crumlin, UK).

Microvascular measurements were conducted in a laboratory at an environmental temperature of $22 \pm 1^{\circ} \mathrm{C}$. Participants were seated with their arms supported at heart level. We assessed microvascular function, as described previously [18], by measuring the volar forearm skin blood flow responses to iontophoresis of acetylcholine (ACh; Sigma-Aldrich, Poole, UK) and sodium nitroprusside (SNP; David Bull Laboratories, Warwick, UK), which are endothelium-dependent and endothelium-independent vasodilators, respectively. At the time of the ethics committee review, due consideration was given to the use of the Sigma ACh preparation, which is a chemical not intended for human use. Iontophoresis is a drug-delivery method, which stimulates the migration of charged ions across the skin non-invasively and without inducing systemic effects.

A single iontophoresis chamber (Moor Instruments, Axminster, UK), consisting of a Perspex ring of internal diameter $20 \mathrm{~mm}$ with a wire electrode running round its inner surface, was fixed to the skin with adhesive tape. The hydrochloride salt of ACh was dissolved in deionised water to produce a $1 \%(w / v)$ solution, and $2 \mathrm{ml}$ of this solution were used to fill the chamber. We use deionised water as the vehicle as we have shown that this does not produce competition for delivery of ions [19]. The positive lead of an iontophoresis controller (MIC2; Moor Instruments) was connected to the chamber, and the negative lead attached to a conductive hydro-gel pad on the wrist, which served as the reference electrode. We used a delivery current of $100 \mathrm{~mA}$, and administered a successive accumulation of 20, 40 and $80 \mathrm{~s}$ doses of Ach, effectively 2, 4 and $8 \mathrm{mC}$ (millicoulombs). Using a current of $100 \mu \mathrm{A}$ and large diameter electrodes does not elicit vasodilatation via nonspecific, galvanic effects, because a low charge density (maximum of $2.5 \times 10^{-2} \mathrm{mC} / \mathrm{mm}^{2}$ ) is generated. The resulting changes in microvascular skin blood flow were assessed at each delivery site using laser Doppler imaging (Moor LDI; Moor Instruments). This technique works by scanning a $2 \mathrm{~mW}$ helium-neon laser across the surface of the skin. Light that is backscattered from moving erythrocytes undergoes a shift in frequency proportional to their velocity, according to the Doppler principle. The resulting colour-coded image represents skin blood flow over the scan area, a relative measure called the laser Doppler flux. A baseline image was recorded at the start. After each iontophoretic delivery, the solution was removed and the chamber dried, and then three images were recorded at $30 \mathrm{~s}$ intervals as the response developed. This was repeated for each dose of ACh. The experiment was repeated at an adjacent site with $1 \%(w / v)$ SNP, dissolved in deionised water. The iontophoresis leads were reversed for SNP because it is negatively charged in solution, and five images were recorded at $45 \mathrm{~s}$ intervals after each dose because SNP takes longer to have an effect on blood flow. The laser Doppler images were analysed using dedicated software (Moor Instruments), and we calculated the median laser Doppler flux over the iontophoresis delivery site for each one. For the response to each dose, the mean of the two stable flux values was taken, and this was divided by the baseline measurement to give a ratio representing the change in flow. This combination of iontophoresis and laser Doppler imaging has been used successfully by us and others in

Table 1 Comparison of patient baseline clinical characteristics

\begin{tabular}{llll}
\hline Variable & Group 1 $(n=27)$ & Group 2 (n=33) & Group 3 (n=31) \\
\hline Male sex & 17 & 15 & 17 \\
Age (years) & $12.7(10.5$ to 14.8$)$ & $14.1(11.7$ to 15.6$)$ & $12.6(11.2$ to 15.4$)$ \\
Carstairs deprivation score ${ }^{\mathrm{a}}$ & $-2.13(-3.73$ to 0.73$)$ & $-1.48(-3.01$ to 1.03$)$ & $-1.76(-3.23$ to 0.93$)$ \\
Ethnicity (non-white) & 1 & 1 & 1 \\
Duration of diabetes (years) & $3.2(1.7$ to 6.7$)$ & $4.8(2.6$ to 8.6$)$ & $5.4(2.9$ to 7.7$)$ \\
BMI SDS & $0.38(-0.44$ to 0.83$)$ & $0.13(-0.55$ to 1.0$)$ & $0.44(0.04$ to 1.04$)$ \\
Systolic BP $(\mathrm{mmHg})$ & $104(102$ to 108$)$ & $106(100$ to 115$)$ & $106(104$ to 115$)$ \\
Diastolic BP $(\mathrm{mmHg})$ & $64(54$ to 70$)$ & $66(58$ to 75$)$ & $66(59$ to 75$)$ \\
HbA $_{1 \mathrm{c}}(\%)$ & $10.1(9.2$ to 11.2$)$ & $9.8(8.6$ to 11.5$)$ & $10.0(9.0$ to 11.4$)$ \\
\hline
\end{tabular}

Values are medians (interquartile range)

${ }^{\text {a }}$ Postcodes were used to obtain the Carstairs deprivation score, which was based on results from the 2001 census. A higher score represents a higher level of deprivation

No significant differences in categorical variables using $\chi^{2}$ or continuous variables using ANOVA were identified

SDS, standard deviation score 
Fig. 1 Flow diagram of patients through the Sweet Talk Study a Enrolment: Five patients were excluded from study - two in temporary foster placements, one moving home, one with a needle phobia, one with severe learning difficulties. b Allocation: One patient withdrew from study after randomisation; of the patients randomised to intensive therapy, 22 patients chose pump therapy, nine patients chose basal bolus therapy and two remained on conventional insulin therapy. c Follow-up: Eight patients changed to basal bolus therapy for clinical reasons; five patients discontinued pump therapy; two changed to basal bolus therapy and three to conventional insulin therapy; one patient moved away from the area during the study

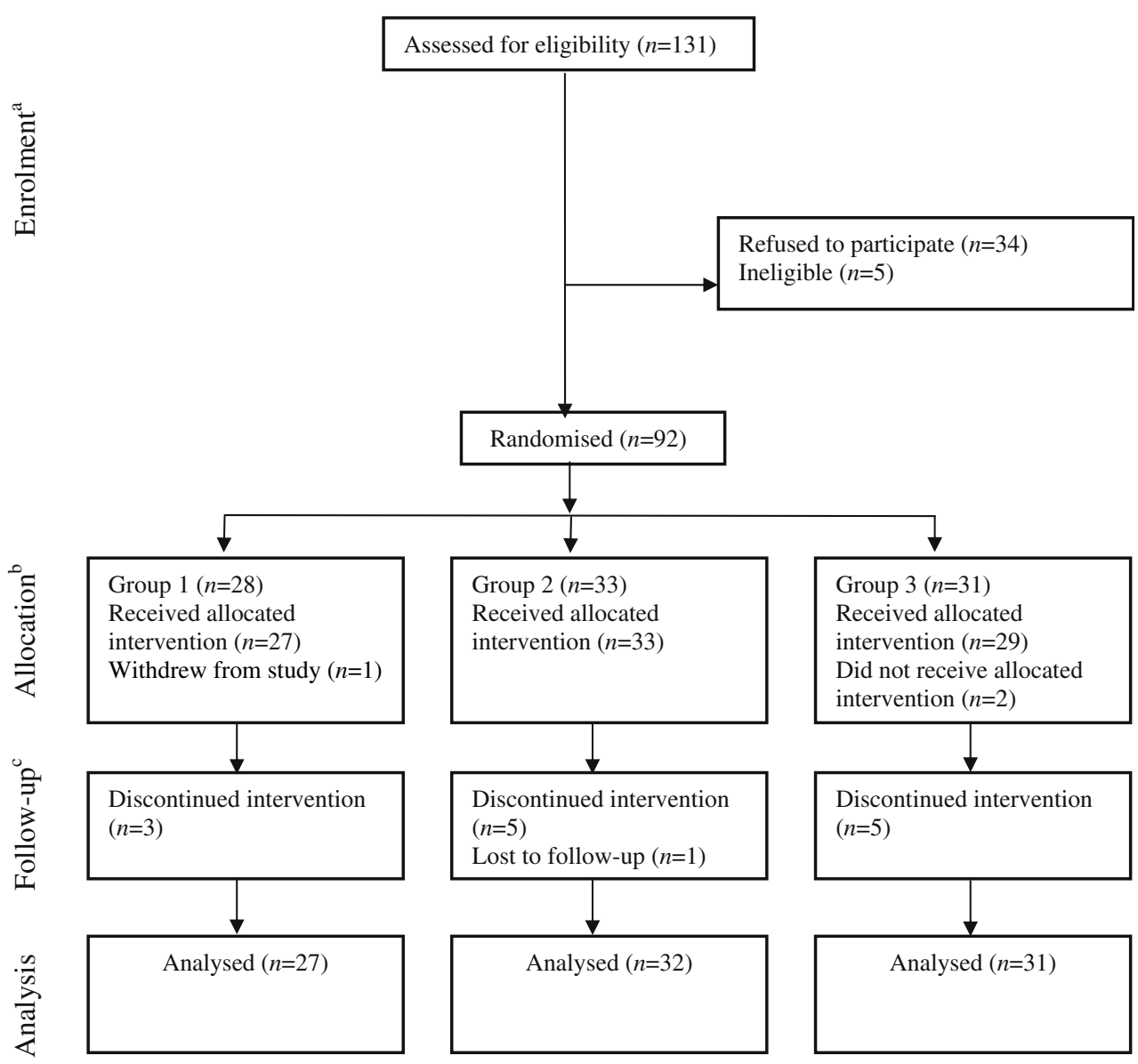

many studies [20, 21], and the good reproducibility of the technique has been confirmed [22, 23]. A recent study showed that skin microvascular responses to iontophoretically applied ACh strongly correlated with flow-mediated dilatation responses in the brachial artery [24].
Statistical analysis Power calculations indicated that a sample of 23 patients per group would detect a difference of $1.7 \%$ in mean $\mathrm{HbA}_{1 \mathrm{c}}(80 \%$ power, two-sided $p$ value $=$ 0.05) mimicking the change seen in the adolescent cohort of the Diabetes Control and Complications Trial of

Table 2 Glycaemic control and microvascular responses (ratio of response over baseline) to ACh and SNP in the three groups before and after intervention

\begin{tabular}{|c|c|c|c|c|c|c|c|}
\hline & \multirow[t]{2}{*}{$\mathrm{HbA}_{1 \mathrm{c}}$} & \multicolumn{3}{|l|}{ Ach } & \multicolumn{3}{|l|}{ SNP } \\
\hline & & $2 \mathrm{mC}$ & $4 \mathrm{mC}$ & $8 \mathrm{mC}$ & $2 \mathrm{mC}$ & $4 \mathrm{mC}$ & $8 \mathrm{mC}$ \\
\hline \multicolumn{8}{|l|}{ CIT } \\
\hline Before & $10.2 \pm 1.6$ & $3.1(2.4-3.8)$ & $3.9(3.19-4.9)$ & $4.9(3.7-5.5)$ & $1.8(1.5-2.5)$ & $3.2(2.6-4.6)$ & $5.2(3.8-6.6)$ \\
\hline After & $10.3 \pm 1.7$ & $3.1(2.0-4.0)$ & $4.2(3.2-5.1)$ & $4.9(4.1-5.3)$ & $1.8(1.3-3.0)$ & $3.1(2.0-5.4)$ & $4.6(3.4-7.2)$ \\
\hline \multicolumn{8}{|c|}{ CIT and Sweet Talk } \\
\hline Before & $9.8 \pm 1.8$ & $3.3(2.0-4.3)$ & $4.5(3.1-5.8)$ & $5.6(3.5-6.3)$ & $1.9(1.6-2.8)$ & $3.1(2.5-4.6)$ & $4.4(3.7-6.5)$ \\
\hline After & $10.1 \pm 1.7$ & $2.9(2.4-3.9)$ & $4.3(3.3-5.3)$ & $5.1(3.9-6.2)$ & $1.9(1.4-2.3)$ & $3.1(2.0-4.3)$ & $4.6(3.8-6.0)$ \\
\hline \multicolumn{8}{|c|}{ ITT and Sweet Talk } \\
\hline Before & $10.2 \pm 2.0$ & $2.7(1.8-3.7)$ & $3.6(2.5-4.5)$ & $4.1(3.5-5.0)$ & $1.6(1.4-2.2)$ & $3.0(2.0-4.1)$ & $4.6(3.0-6.0)$ \\
\hline After & $9.2 \pm 2.2$ & $2.7(1.9-3.6)$ & $4.1(3.1-5.1)$ & $5.2(4.1-6.1)$ & $1.8(1.4-2.4)$ & $3.2(1.8-4.2)$ & $4.5(3.2-5.8)$ \\
\hline
\end{tabular}

Values for $\mathrm{HbAl}_{\mathrm{c}}$ are means $\pm \mathrm{SD}$, the remainder are medians (interquartile range)

Responses to ACh were significantly greater after intervention with ITT and Sweet Talk compared with the other two groups at all concentrations tested 
9.8-8.1\%. Data for microvascular responses were not normally distributed and were therefore $\log _{10}$-transformed to achieve normality. Differences in the dose-response to ACh and SNP before and after each particular intervention were tested across the three groups using a three-way ANOVA model for repeated measures. In addition, comparisons between the absolute changes (before vs after) in microvascular responses across the three different groups were compared using ANOVA. Post hoc analysis used the Bonferroni test. Correlations were performed using the Spearman rank method. In all cases, significance was acknowledged if the probability of a type 1 error was $<5 \%$ (i.e. $p<0.05$ ). With respect to microvascular function, the power calculation is based on repeat measurements at two time-points at least 2 months apart in 44 individuals. The SD of the change for the ACh response (defined as the ratio of response over baseline) is 0.80 . Therefore we are able to detect a difference of $0.5 \mathrm{SDs}$ (which equates to a $13.4 \%$ change) with $80 \%$ power at $p<0.05$ change using a paired comparison in 25 patients. We have previously shown that $n=29$ patients are sufficient to detect differences of $14 \%$ between 11- to 14-year-old children with low and high $2 \mathrm{~h}$ glucose levels [18]. Additionally we have shown that $n=28$ is sufficient to show improvements of $29 \%$ in normal individuals before and after intervention [25].

The blood results were also not normally distributed and Wilcoxon signed rank tests were performed to compare differences between the levels at visit 1 and visit 2 . The values in Table 4 are the median levels and the 25th and 75th percentiles.

Outcome data were compared with baseline. Data analysis was performed on an intention-to-treat basis. The authors had full access to the data and take responsibility for its integrity. All authors have read and agree to the manuscript as written.

\section{Results}

Ninety-two patients were recruited over a 5 month period from October 2002 and each patient was enrolled in the study for 1 year. There were no significant differences in baseline demographic or clinical characteristics between the patients who declined to participate in the study and those who enrolled, and there were no differences between the groups at baseline (Table 1). One patient withdrew from group 1 after randomisation, and one patient from group 2 moved from the area. Three patients from group 1, and five from group 2 were changed to ITT during the study for clinical reasons. Of the 31 patients allocated to group 3, 22 chose an insulin pump and nine chose basal bolus therapy. Of these, two patients remained on CIT, and three patients changed from pump therapy to CIT during the year of the study. All participants were analysed on an intention-totreat basis. Flow through the study is illustrated in Fig. 1.

Glycaemic control deteriorated in patients on CIT, but improved significantly in patients allocated to IIT ( $p=0.007$, ANOVA). IIT (group 3) was associated with significantly greater improvements in E-selectin, a key endothelial marker, elevated levels of which have been linked to future

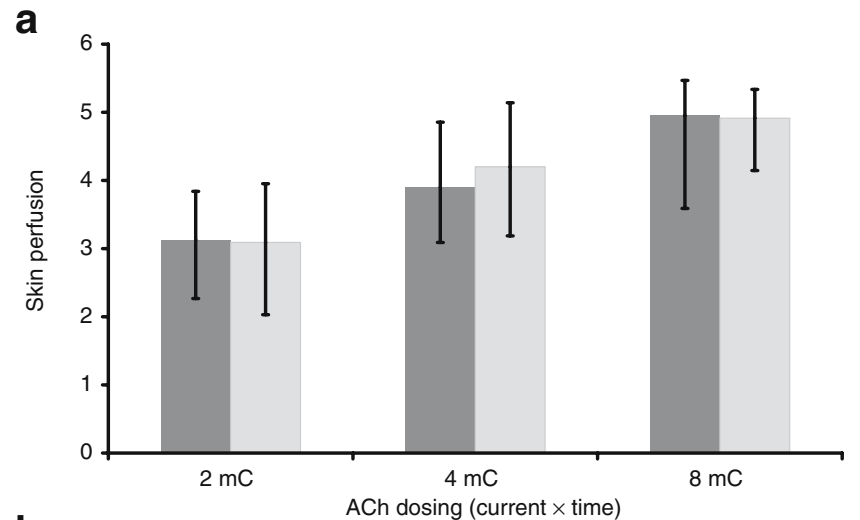

b

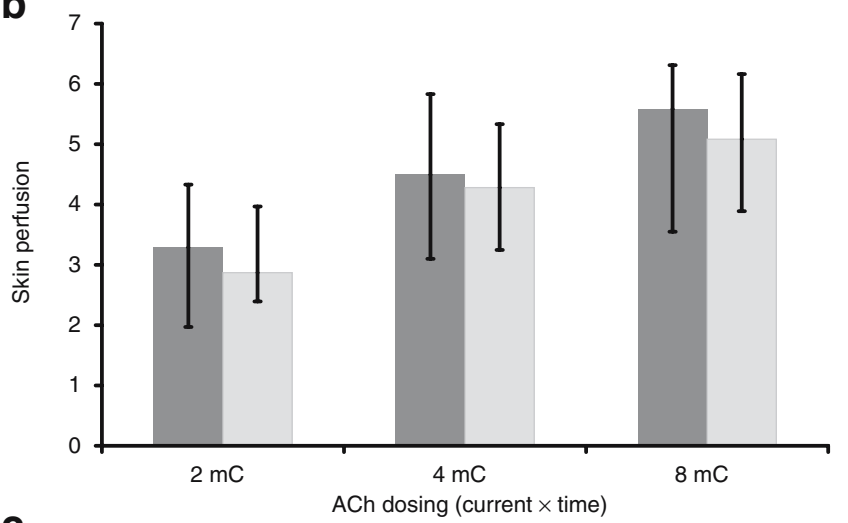

C

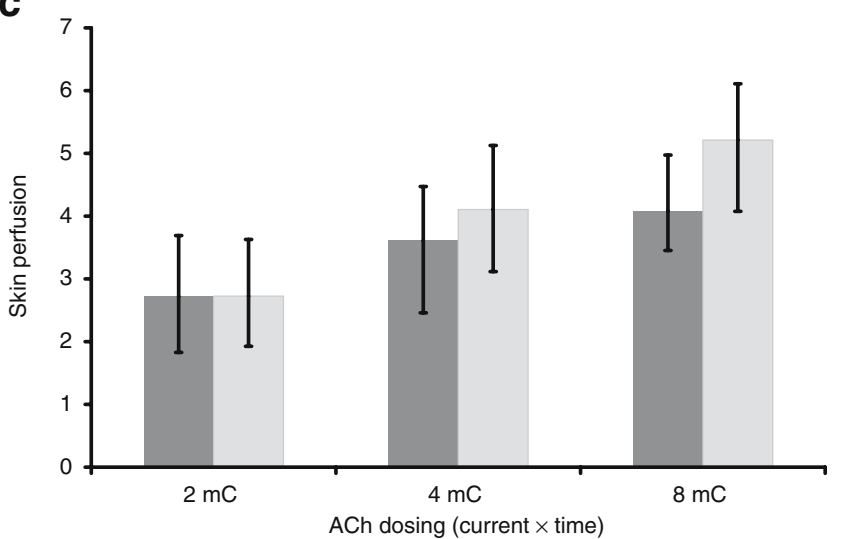

Fig. 2 Microvascular responses (skin perfusion expressed as the ratio of response over baseline) to ACh before (dark grey columns) and after intervention (light grey columns) in CIT (a), CIT and Sweet Talk (b), and IIT and Sweet Talk (c) groups. Responses to ACh were significantly greater after intervention in the IIT and Sweet Talk group compared with the other two groups $(p=0.017$, ANOVA comparing the improvements in skin perfusion in response to intervention between the three groups). Values are medians (interquartile range) 
Table 3 Before vs after changes in microvascular responses to ACh and SNP in the three groups

\begin{tabular}{|c|c|c|c|c|c|c|}
\hline & \multicolumn{3}{|l|}{$\mathrm{ACh}$} & \multicolumn{3}{|l|}{ SNP } \\
\hline & $2 \mathrm{mC}$ & $4 \mathrm{mC}$ & $8 \mathrm{mC}$ & $2 \mathrm{mC}$ & $4 \mathrm{mC}$ & $8 \mathrm{mC}$ \\
\hline CIT & $0.06(-1.00$ to 1.14$)$ & $0.21(-0.61$ to 1.31$)$ & $0.09(-0.83$ to 1.08$)$ & $0.13(-0.31$ to 0.70$)$ & $-0.13(-1.01$ to 1.23$)$ & $0.18(-1.46$ to 1.07$)$ \\
\hline $\begin{array}{l}\text { CIT and } \\
\text { Sweet Talk }\end{array}$ & $-0.09(-0.92$ to 0.50$)$ & $-0.16(-0.91$ to 1.16$)$ & $0.08(0.41-0.99)$ & $-0.37(-0.91$ to 0.38$)$ & $-0.75(-1.80$ to 0.83$)$ & -0.54 ( -2.67 to 1.32$)$ \\
\hline $\begin{array}{l}\text { IIT and } \\
\text { Sweet Talk }\end{array}$ & $-0.14(-0.77$ to 0.77$)$ & $0.38(-0.32$ to 1.35$)$ & $0.78(0.08-1.65)^{*}$ & $0.06(-0.52$ to 0.74$)$ & $-0.11(-0.70$ to 1.29$)$ & 0.01 ( -0.83 to 1.61$)$ \\
\hline
\end{tabular}

Values are medians (interquartile range). Positive values indicate an increase in microvascular response

Microvascular changes were greater in the ITT and Sweet Talk group compared with the other two groups ( $p=0.003$, ANOVA)

${ }^{*} p<0.05$ post hoc test for differences at the highest ACh dose

cardiovascular events [26]; this was statistically significant $(p<0.0001)$ over the CIT (group $1 p=0.026$; group $2 p=$ 0.053 , ANOVA).

Table 2 shows the baseline values for microvascular responses to ACh and SNP in the three groups. There was a trend for microvascular responses to be higher in group 2 (CIT+text message support) compared with responses in the other two groups ( $p=0.094$ for ACh and $p=0.03$ for SNP), which were accounted for by differences in the male to female ratios. Correcting for sex showed that the trends for $\mathrm{ACh}$ and SNP responses to be greater in group 2 were no longer apparent ( $p=0.226$ for ACh and $p=0.11$ for SNP).

There were no significant correlations between any of the clinical variables, such as duration of diabetes, $\mathrm{HbA}_{1 \mathrm{c}}$, insulin dose) and microvascular responses to ACh and SNP.

Table 2 and Fig. 2 show that in comparison with the changes in microvascular responses to ACh in groups 1 and 2, group 3, who received IIT and text messaging support, showed a significant improvement in microvascular response to ACh ( $p=0.017$, ANOVA). At the two highest doses of $\mathrm{ACh}$, microvascular responses increased by 11.4 and $14.8 \%$ compared with pre-intervention values, respectively. In addition, comparing the absolute before vs after differences in ACh responses among the three groups showed that the IIT group had the greatest improvement compared with the other two groups ( $p=0.003$, ANOVA; Table 3$)$. There was no significant change in vascular responses to SNP before and after intervention in any of the groups. There was no correlation between the improvement in vascular responses to $\mathrm{ACh}$ and the lowering of $\mathrm{HbA}_{1 \mathrm{c}}$ levels in group 3 .

Table 4 shows the results of markers of endothelial dysfunction and activation. It was not possible to obtain the full volume of blood required for these tests from all patients, so the number of patients for whom data were available is also indicated. There were no significant differences in lipid profiles (cholesterol, triacylglycerols, HDL or LDL) between the groups at baseline or end of the study.

\section{Discussion}

Patients receiving intensive diabetes management, comprising ITT and Sweet Talk intensive support, showed significant improvements in glycaemic control compared with the groups allocated to CIT regimens. The overall reduction of $1.1 \%$ when adjusted for baseline (from 10.2 to $9.2 \%$ ) is of clinical significance as it is associated with approximately a $40 \%$ reduction in risk of developing microvascular complications [27].

Table 4 Lipid profiles and vascular markers at baseline and end of the study

\begin{tabular}{llll}
\hline Test $(n)$ & Baseline & End of study & $p$ value \\
\hline HbA $_{1 \mathrm{c}}(\%)$ & & & \\
Group 1 (27) & $10.1(9.2-11.2)$ & $10.4(9.2-11.6)$ & 0.034 \\
Group 2 (33) & $9.8(8.6-11.5)$ & $10.3(9.0-11.2)$ & 0.75 \\
Group 3 (31) & $10.0(9.0-11.4)$ & $9.2(7.2-10.6)$ & $<0.001$ \\
Cholesterol (mmol/1) & & \\
Group 1 (26) & $4.8(4.1-5.4)$ & $4.6(4.1-5.1)$ & 0.864 \\
Group 2 (20) & $4.5(4.4-5.7)$ & $4.7(4.2-5.3)$ & 0.653 \\
Group 3 (25) & $5.0(4.6-5.6)$ & $5.0(4.5-5.2)$ & 0.456 \\
Triacylglycerols (mmol/1) & & \\
Group 1 (26) & $0.8(0.5-1.4)$ & $0.9(0.6-1.4)$ & 0.137 \\
Group 2 (20) & $0.73(0.55-0.9)$ & $0.8(0.6-1.0)$ & 0.831 \\
Group 3 (25) & $0.68(0.6-0.97)$ & $0.75(0.6-1.1)$ & 0.475 \\
HDL (mmol/1) & & & \\
Group 1 (26) & $1.43(1.22-1.81)$ & $1.36(1.29-1.59)$ & 0.090 \\
Group 2 (20) & $1.63(1.38-1.86)$ & $1.59(1.41-1.98)$ & 0.130 \\
Group 3 (25) & $1.60(1.49-1.83)$ & $1.69(1.54-1.82)$ & 0.276 \\
LDL (mmol/l) & & & \\
Group 1 (26) & $1.75(1.42-2.04)$ & $1.59(1.36-1.99)$ & 0.242 \\
Group 2 (20) & $1.71(1.51-2.27)$ & $1.77(1.48-2.09)$ & 0.522 \\
Group 3 (25) & $1.95(1.70-2.15)$ & $1.85(1.73-2.13)$ & 0.117 \\
E-selectin (ng/ml) & & & \\
Group 1 (27) & $93.4(73.8-121.4)$ & $82.3(68.8-103.5)$ & 0.026 \\
Group 2 (22) & $80.4(56.5-104.1)$ & $68.4(39.2-92.0)$ & 0.053 \\
Group 3 (29) & $87.8(58.1-109.3)$ & $65.0(41.6-87.0)$ & $<0.0001$ \\
\hline
\end{tabular}

Values are medians (interquartile range) 
Patients treated with ITT showed significant improvements in E-selectin levels, which confirms findings of a previous study, which showed that improvements in E-selectin correlate with improvements in glycaemic control [28]. The ITT and Sweet Talk groups showed significant improvements in endothelial-dependent (ACh) microvascular responses, which were independent of improvements in glycaemic control, possibly suggesting that intensive therapy may modify endothelial dysfunction by additional means. In our view this is consistent with results from the Diabetes Control and Complications Trial, where it has been suggested that the ITT itself, compared with conventional therapy, confers an additional benefit over and above any improvement in glycaemic control [29]. The mechanisms of endothelial damage in diabetes are incompletely understood at present, but it seems unlikely that ambient glucose levels are a sole factor. Insulin modulates endothelial function including vascular reactivity, thrombotic processes and inflammation. Therefore ITT may confer its advantages by delivering a more physiological insulin profile together with the reduction in glycaemic excursions. Other factors including smoking habit and hypertension may have influenced vascular outcome, but these data are not available.

Future research should aim to determine whether vascular function is influenced by the mechanism of intensive insulin delivery (pump therapy or basal bolus therapy). Furthermore, longitudinal studies are needed to determine whether the improvements in glycaemic control can be maintained, and whether the improvements in markers of endothelial function and vascular control translate into a reduction in the development of the micro- and macrovascular complications of diabetes.

In conclusion, these novel findings suggest that intensive diabetes management improves metabolic control, endothelial function and microvascular reactivity in young people with type 1 diabetes. This has important consequences for future management of these children. Confirmed surrogate markers for future cardiovascular events are favourably altered by such an intensive regimen. The improvements in vascular markers appear to occur independently of improvements in glycaemic control, suggesting that IIT confers additional vascular protection, possibly by delivering a more physiological insulin profile, which is important because of the reduction in glycaemic excursion, and because insulin is a vasodilator with potent anti-inflammatory effects. This study supports the early introduction of intensive therapy in young people with diabetes, as reducing the antecedents of angiopathy will translate into significant health advantages in adulthood.

Acknowledgements This project was supported by Diabetes UK and the Chief Scientist Office, Scotland, Grant number CZB/4/36. F. Khan and J. J. F. Belch are supported by the Sir John Fisher
Foundation. The Sweet Talk text messaging intervention was supported by Orange and The Sea, and Minimed Medtronic provided the insulin pumps for the study. We are also grateful to M. McLaren for her help with laboratory analyses.

Duality of interest S. Greene has received honoraria and lecture fees from Medtronic. The other authors declare that there is no duality of interest associated with this manuscript.

\section{References}

1. Laing SP, Swerdlow AJ, Slater SD et al (1999) The British Diabetic Association Cohort Study, 1: all course mortality in patients with insulin-treated diabetes mellitus. Diabet Med 16:466-471

2. Rayman G, Williams SA, Spencer PD (1986) Impaired microvascular hyperaemic response to minor skin trauma in type 1 diabetes. Clin Res 292:1295-1298

3. Wilson S, Jennings P, Belch J (1992) Detection of microvascular impairment in type 1 diabetes by laser Doppler flowmetry. Clin Physiol 12:197-210

4. World Health Organisation Expert Committee on Diabetes Mellitus (1980) 2nd Report. World Health Organ Tech Rep Ser 646:9-14

5. Diabetes Control and Complications Trial Research Group (1994) Effect of intensive diabetes treatment on the development and progression of long-term complications in adolescents with insulin-dependent diabetes mellitus: Diabetes Control and Complications Trial. J Pediat 125:177-188

6. Jones SL, Close CF, Mattock MB (1989) Plasma lipid and coagulation factor concentrations in insulin dependent diabetics with microalbuminuria. BMJ 298:487-490

7. Pearson JD (1994) Vessel wall interactions regulating thrombosis. Br Med Bull 50:776-788

8. Elhadd TA, Kennedy G, Hill A et al (1999) Abnormal markers of endothelial cell activation and oxidative stress in children, adolescents and young adults with type 1 diabetes with no clinical vascular disease. Diabetes Metab Res Rev 15:405-411

9. Khan F, Elhadd TA, Greene SA, Belch J (2000) Impaired skin microvascular responses in children, adolescents and young adults with type 1 diabetes mellitus. Diabetes Care 23:215-220

10. McLaren M, Elhadd TA, Greene SA, Belch J (1999) Elevated plasma vascular endothelial cell growth factor and thrombomodulin in juvenile diabetic patients. Clin Appl Thromb Hemost 5:21-24

11. National Institute for Clinical Excellence (2004) Type 1 diabetes in children and young people. NICE, London

12. Scottish Intercollegiate Guidelines Network (2001) Scottish Intercollegiate Guidelines Network management of diabetes. No. 55. SIGN, Edinburgh

13. Franklin V, Waller A, Pagliari C, Greene S (2003) 'Sweet Talk': text messaging support for intensive insulin therapy for young people with diabetes. Diabetes Technol Ther 5:991-996

14. Franklin V, Waller A, Pagliari C, Greene S (2006) 'Sweet Talk' text-messaging support for young people with diabetes. Diabet Med 23:1332-1338

15. Shammin-Uzzman QA, Pfenninger D, Kehrer C et al (2002) Altered cutaneous microvascular responses to reactive hyperaemia in coronary artery disease: a comparative study with conduit vessel responses. Clin Sci 103:267-273

16. Ijzerman RG, De Jonagh RT, Beijk MAM et al (2003) Individuals at increased coronary heart disease risk are characterized by an impaired microvascular function in skin. Eur $\mathrm{J}$ Clin Invest $33: 536-542$ 
17. Mortensen HB (1998) Insulin management and metabolic control of type 1 diabetes mellitus in childhood and adolescence in 18 countries. Diabet Med 15:752-759

18. Khan F, Green FC, Forsyth JS, Greene SA, Morris AD, Belch J (2003) Impaired microvascular function in normal children: effects of adiposity and poor glucose handling. J Physiol 551:705-711

19. Khan F, Newton DJ, Smyth EC, Belch JJF (2004) The influence of vehicle resistance on transdermal iontophoretic delivery of acetylcholine and sodium nitroprusside in humans. J Appl Physiol 97:883-887

20. Martin H, Hu J, Gennser G, Norman M (2000) Impaired endothelial function and increased carotid stiffness in 9-year-old children with low birthweight. Circulation 2739-2744

21. Morris SJ, Shore AC, Tooke JE (1995) Responses of the skin microcirculation to acetylcholine and sodium nitroprusside in patients with NIDDM. Diabetologia 38:1337-1344

22. Kubli S, Waeber B, Dalle-Ave A, Feihl F (2000) Reproducibility of laser Doppler imaging of skin blood flow as a tool to assess endothelial function. J Cardiovasc Pharmacol 36:640-648

23. Newton DJ, Khan F, Belch J (2001) Assessment of microvascular endothelial function in human skin. Clin Sci 101:567-572

24. Hansell J, Henareh L, Agewall S, Norman M (2004) Non-invasive assessment of endothelial function - relation between vasodilatory responses in skin microcirculation and brachial artery. Clin Physiol Funct Imaging 24:317-322

25. Khan F, Elherik K, Bolton-Smith C et al (2003) The effects of dietary fatty acid supplementation on endothelial function and vascular tone in healthy subjects. Cardiovasc Res 59:955962

26. Blann AD, McCollum CN, Lip GY (2002) Relationship between plasma markers of endothelial cell integrity and the Framingham cardiovascular disease risk-factor scores in apparently healthy individuals. Blood Coagul Fibrinolysis 13:513-518

27. The Diabetes Control and Complications Trial (1996) The absence of a glycaemic threshold for the development of long-term complications: the perspective of the diabetes control and complications trial. Diabetes 45:1289-1298

28. Troseid M, Lappegard KT, Mollnes TE, Arnesan H, Sejeflot I (2005) Changes in serum level of E-selectin correlate to improved glycaemic control and improved obesity in subjects with the metabolic syndrome. Scand J Clin Lab Invest 65:283-290

29. Schaumberg DA, Glynn RJ, Jenkins AJ et al (2005) Effect of intensive glycaemic control on levels of markers of inflammation in type 1 diabetes mellitus in the diabetes control and complications trial. Circulation 111:2446-2453 